\title{
Defining a Reference Architecture for Transformed Health Ecosystems
}

\author{
Bernd BLOBEL ${ }^{\mathrm{a}, \mathrm{b}, \mathrm{c}, 1}$ and Kenneth RUBIN ${ }^{\mathrm{d}}$ \\ ${ }^{a}$ Medical Faculty, University of Regensburg, Germany \\ ${ }^{\mathrm{b}}$ eHealth Competence Center Bavaria, Deggendorf Institute of Technology, Germany \\ ${ }^{\mathrm{c}}$ First Medical Faculty, Charles University of Prague, Czech Republic \\ ${ }^{\mathrm{d}}$ University of Utah, Salt Lake City, Utah, USA
}

\begin{abstract}
The paper discusses requirements and solutions for design and management of transformed health ecosystems. After introducing related definitions with reference to the transformation of health to P5 medicine, basics on systems, knowledge representation and management as well as system development processes and their formal representation/modelling from the perspectives of systems theory, theory of knowledge, languages and grammars are considered in some detail. As result, the ISO 23903 reference architecture is shortly introduced and compared with other existing approaches and standards.
\end{abstract}

Keywords. Ecosystems, P5 medicine, reference architecture, concepts, knowledge representation, modeling, integration, standards

\section{Introduction}

An ecosystem is a system or network of living and nonliving interconnecting and interacting elements to meet specific objectives. Thereby, it explicitly considers related intentions and actions of involved business actors. A system can be analyzed and described, using a black box or a white box approach. The black box approach just considers the input-output relations of a system, while the white box approach specifies structure, functions and relations of all components constituting that system.

Personalized, preventive, predictive and participative precision (P5) medicine is the latest move in health ecosystem transformation [1,2]. The transformed health ecosystem comprises multiple disciplines, representing (parts of) the reality from the elementary particle up to the universe, or from a health perspective, from cell components through cells, tissues, organs, and individuals up to the society. It integrates, e.g., medicine and life sciences, natural and social sciences, bioinformatics, economy, ecology, and engineering, but also research and practice. It systematically enhances empiric and evidence-based medicine to systems medicine by understanding the course of diseases according to the individual health status, context and conditions of the subject of care including their prediction as well as appropriate prevention and treatment. That way, it advances the health system from a black box to a white box representation. Stakeholders from many different knowledge as well as policy domains with specific perspectives on

${ }^{1}$ Corresponding Author. Bernd Blobel, PhD, FAACMI, FACHI, FHL7, FEFMI, FIAHSI, Professor, Medical Faculty, University of Regensburg, Germany; Email: bernd.blobel@klinik.uni-regensburg.de 
the system, using their methodologies, terminologies and ontologies at different levels of maturity, knowledge, skills and experiences, provide this highly interdisciplinary approach. Intersections between, and needed integration of, those diversities present inherent challenges, driven both by the lack of shared expertise and by inherent miscommunications across disciplines, resulting in incompatible or incomplete solutions that frequently fail or fall short of their potential.

A reference architecture is commonly defined as a document or set of documents that provides recommended structures, functions and integrations of IT products and services, i.e. ICT specifications and resources represented using ICT ontologies, to form a solution. It embodies accepted industry best practices, thereby usually focusing on specific technologies. A reference architecture for transformed health ecosystems has to combine both the representation of the multidisciplinary ecosystem architecture and the development process of software enabling or supporting the ecosystem's business objectives. The challenge is to represent and integrate the knowledge of the different domains involved as well as the related enterprise and process models, their informational representations, and finally the implementable artifacts in one schema or framework.

The paper considers ecosystems and especially the transformed health ecosystem, but also the system evolution or system development process from different perspectives, deploying related theories, methodologies and representation styles to define a multidomain ecosystem's reference architecture. Here, systems theory, theory of knowledge, cognitive sciences and philosophy, theory of languages including their abstraction, expressivity and scope-specific grammars, system development processes, modeling good practices, enterprise architectures, information and data modeling, etc., have to be named.

\section{Methods}

Systems theory defines a system as a set of interrelated elements (components) differentiated from the system's environment it interacts with. A system's architecture describes its components, their functions and relations, i.e., the system's structure and behavior. Therefore, the transformed health ecosystem is represented by knowledge, functions and operations of its subsystems or domains and the relations and dependencies among them, so forming a system of systems. [3]

To enable flexibility and re-usability, a reference architecture should be a composite, defined along different levels of abstraction or views according to ISO/IEC 10746, ISO/IEC/IEEE 42010 or the Rational Unified Process (RUP). Another reference architecture is OMG's Mode Driven Architecture (MDA) with its computationindependent models, platform-independent models and platform-specific models. For representing the different views or models, different languages with grammars at different level of generative power, expressivity and constraints ranging from domainspecific, context-aware natural languages with high generative power and less constraints through context-sensitive languages up to regular ones such as programming languages, must be deployed to develop and implement the intended ecosystem. To enable knowledge-driven, cross-domain interoperability and system integration as required for transformed health ecosystems, the development process must be combined with the multi-domain representation of the system. Just ISO 23903 Interoperability and integration reference architecture - Model and framework defines such approach. 


\section{Systems Representation}

Structurally, the system is composed of subsystems, sub-subsystems, etc., according to the granularity levels in the process of components' composition/decomposition. The structural representation of the ecosystem follows the set theory, while the architectural representation of the ecosystem's concept world guiding its structure, function and behavior requires a constructive approach to mathematics using mathematical logics and type theory or in general universal type theory and universal logics. Barendregt introduced a generalization of the simply typed $\lambda$-calculus towards a three-dimensional calculus of construction, where each dimension represents another kind of dependency between terms and types, summarized as concepts [4].

For grouping interrelated elements in the sense of the domain's architectural compositions, the parametrized Barendregt Cube [5] has been deployed and transformed into a system-theoretical representation. The outcome is a cube consisting of three dimensions according to the Generic Component Model [6]: the domain dimension, the system's granularity level dimension, and the system's evolution level.

\section{Knowledge Representation}

Doerner defines domain knowledge as a set of reproducible and reliable models of a domain, representing that domain's perspective on reality to facilitate reasoning, inferring, or drawing conclusions. Those models can be repeatable formulated and justified in the domain of discourse [7]. The knowledge models represent information combined with experience, context, interpretation, and reflection to enable actions and decisions [8]. We have to distinguish three levels of knowledge representation: a) epistemological level (domain-specific modeling), b) notation level (formalization, concept representation), and c) processing level (computational, implementations).

For representing the concept space of a domain, the related domain ontology providing a formal explicit specification of a shared conceptualization of a domain of interest is used. Therefore, it describes an ordering system of entities of a domain with their concepts, functions, and relations, thereby instantiating the architectural components of the considered ecosystem.

\section{Good Modeling Practices}

A model conceptually represents some parts or aspects of reality, i.e. empirical objects, phenomena and processes, in an unambiguous, abstract, and at best formal way. Because of specific audiences resulting in specific requirements and modeling goals, but also due to behavioral, conceptual or contextual differences (e.g. knowledge, experiences, skills, etc. with the aforementioned consequences), two models of the same phenomenon may be quite different ambiguous. This effect gets even stronger, when the models represent views expressed through more restrictive and expressive, and therefore less powerful and context-free languages and related grammars. For overcoming related problems and guaranteeing conceptual integrity when integrating different KR models, good modeling design principles such as orthogonality (not linking independent elements, separate concerns), generality (austerity), parsimony (avoiding irrelevance), and propriety (appropriateness) [9] must be met. To get this, the relevant stakeholders shall define the 
provided view of the model as well as the way of structuring and naming the concepts of the problem space according to the aforementioned principles. Furthermore, data models at all levels (very high level, high level, logical, physical) [10], the related dimensions of modeling (knowledge space, knowledge, information, data) [11] and the different information level (external, conceptual, logical, physical) [12] must be developed and included. For deciding on the correctness of relations and dependencies at one level of modeling, we have to move up to a higher modeling level and finally up to the real-world knowledge space. For more details, see [3].

\section{The ISO 23903 Interoperability and Integration Reference Architecture}

ISO 23903 is a mathematically, logically and philosophically founded system-oriented, architecture-centric, ontology-based reference architecture, which extends the viewpoints defined by ISO 42010, ISO 10746, or RUP by a real-world business view. Furthermore, it details OMG's Model Driven Architecture (MDA) Computation Independent Modeling (CIM) by adding to the view dimension, a component composition dimension and a domain dimension, consequently turning the aforementioned ISO specification into a three-dimensional reference model (Figure 1).

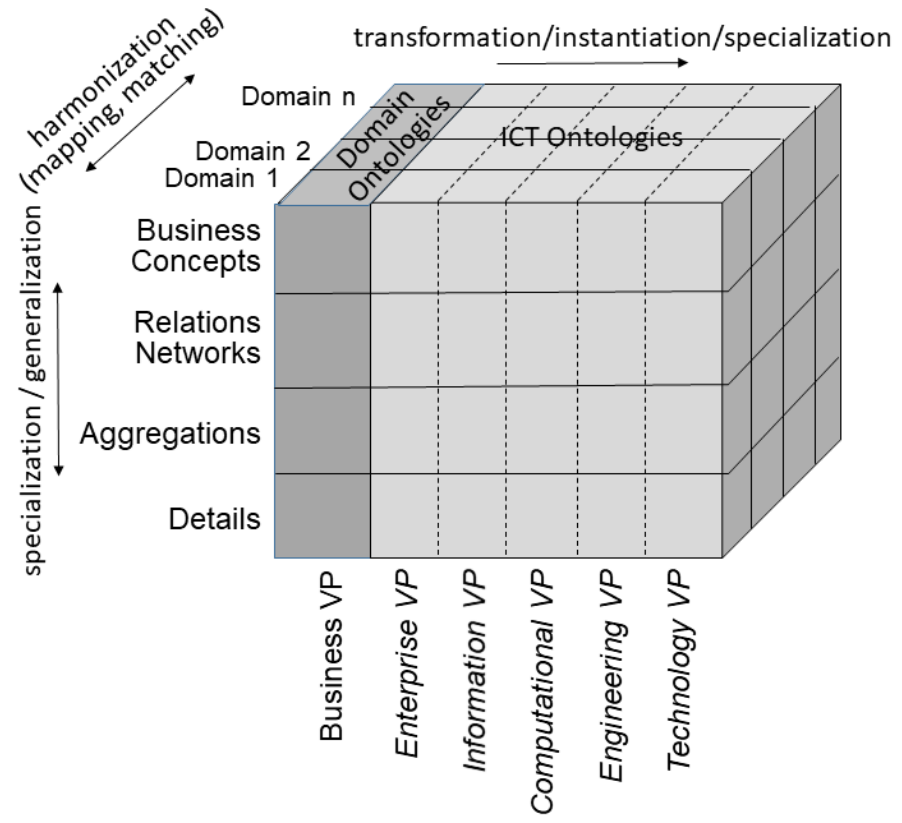

Figure 1. ISO DIS 23903 Mandatory Model and Framework.

\section{Discussion}

The presented ISO 23903 Reference Architecture enables cross-domain concept/knowledge mapping and sharing, supported by ISO 21838 Top Level Ontologies, as well as integration of, and interoperability between, independently developed 
specifications and related products by transferring/re-engineering proprietary into standardized and domain-specific concept representations without requiring any revisions of the original standards/specifications.

Beside the already discussed and with ISO 23903 interrelated standards ISO/IEC/IEEE 42010, ISO/IEC 10746 ODP-RM and OMG's MDA, there are several other architectural approaches or reference architectures established as international standards or specifications of consortia like The Open Group and others. In the context of this paper, the Zachman Framework for Information Systems Architecture, later on generalized towards the Zachman Framework for Enterprise Architecture, but also the US Federal Enterprise Architecture Framework (FEAF) and The Open Group Architecture Framework (TOGAF) have to be mentioned here. An overview on those approaches and their unified representation for integration acc. to ISO 23903 can be found in [13]. The newest project on this matter is the ISO/IEC JTC 1/AG 8 "Meta Reference Architecture and Reference Architecture for Systems Integration".

From the modeling perspective, most if not all those models and approaches miss one or more of the necessary knowledge representation levels presented in Section 3: epistemological level (domain-specific modeling), notation level (formalization, concept representation), and processing level (computational, implementations).

ISO 23903 enables the harmonization and integration of existing architecture models and frameworks as well as reference architectures (RAs) named before, but also domainspecific ones such as IoT RA, Industry 4.0 RA, Smart Cities RA, etc. Ongoing projects frequently ignore the presented principles, challenges and limitations by trying just to aggregate "related" information or data models or to perform term-based mappings. Because of its foundations, the provided reference architecture is sustainable, also enabling the adoption to, and inclusion of, newer techniques.

\section{References}

[1] Blobel B. Challenges and Solutions for Designing and Managing pHealth Ecosystems. Front. Med. 2019; 6:83.

[2] Blobel B, Ruotsalainen P. Healthcare Transformation Towards Personalized Medicine - Chances and Challenges. Stud Health Technol Inform. 2019;261,3-21.

[3] Blobel B, Ruotsalainen P, Oemig F. Why Interoperability at Data Level Is Not Sufficient for Enabling pHealth? Stud Health Technol Inform. 2020;273,3-19.

[4] Kamareddine F, Laan T, Nederpelt R. A Modern Perspective on Type Theory. New York: Kluwer Academic Publishers; 2004.

[5] Kamareddine F, Laan T, Nederpelt R. Refining the Barendregt Cube using Parameters. LNCS 2001,2024, 375-389.

[6] Blobel B. Architectural approach to eHealth for enabling paradigm changes in health. Methods Inf Med 2010; 49,2: 123-134.

[7] Doerner H. Knowledge Representation. Ideas - Aspects - Formalisms. In: Grabowski J, Jantke KP, Thiele H (Edrs). Foundations of Artificial Intelligence. Berlin: Akademie-Verlag; 1989.

[8] Davenport TH, De Long DW, Beers MC. Successful Knowledge Management Projects. Sloan Management Review; Winter 1998; 39, 2; ABI/INFORM Global, pp. 43-57.

[9] Lankhorst M, et al., Enterprise Architecture at Work. The Enterprise Engineering Series. Berlin Heidelberg: Springer-Verlag; 2009.

[10] Hoberman S, Burbank D and Bradley C. Data Modeling for the Business: A Handbook for Aligning the Business with IT using High-Level Data Models. Bradley Beach, NJ: Technics Publications, LLC.; 2009.

[11] Krogstie J. Business Information Systems Utilizing the Future Internet. LNBIP 2011;90:1-18.

[12] Ponniah P. Data Modeling Fundamentals. Hoboken, New Jersey: A John Wiley \&Sons, INC.; 2007.

[13] Blobel B, Oemig F. The Importance of Architectures for Interoperability. Stud Health Technol Inform. 2015;211:18-56. 